\title{
Landmark Detection Based on Sensor Fusion for Mobile Robot Navigation in a Varying Environment
}

\author{
TaeSeok Jin ${ }^{*}$ Hyun-Sik Kim ${ }^{* *}$ and Jong-Wook Kim ${ }^{* * *}$ \\ * Dept. of Mechatronics Engineering, DongSeo University, Busan, 617-716, Korea \\ ${ }^{* *}$ Dept. of Robot System Engineering, TongMyong University, Busan, 608-711, Korea \\ ***Dept. of Electronics Engineering, Dong-A University, Busan, 604-714, Korea
}

\begin{abstract}
We propose a space and time based sensor fusion method and a robust landmark detecting algorithm based on sensor fusion for mobile robot navigation. To fully utilize the information from the sensors, first, this paper proposes a new sensor-fusion technique where the data sets for the previous moments are properly transformed and fused into the current data sets to enable an accurate measurement. Exploration of an unknown environment is an important task for the new generation of mobile robots. The mobile robots may navigate by means of a number of monitoring systems such as the sonar-sensing system or the visual-sensing system. The newly proposed, STSF (Space and Time Sensor Fusion) scheme is applied to landmark recognition for mobile robot navigation in an unstructured environment as well as structured environment, and the experimental results demonstrate the performances of the landmark recognition.
\end{abstract}

Key Words : Sensor fusion, Mobile robot, Measurement, Image processing, Navigation, Global histogram.

\section{Introduction}

In recent years interests have been growing in the synergistic use of multiple sensors to increase the capabilities of intelligent machines and systems [1]. For these systems to use multiple sensors effectively, a strategy to integrate the information provided by these sensors into the operations of the system is necessary. While in many multi-sensor systems the information from each sensor serves as a separate input to the system, the actual combination or fusion of information prior to its use in the system has been a particularly active area of research. Typical applications that can benefit from the use of multiple sensors are industrial tasks like an assembly, a military command and control for battlefield management, mobile robot navigation, multi-target tracking, and aircraft navigation. In all of these applications, the system needs to intelligently interact with the human and operates in an unstructured environment with the human operator's assistant[2,3].

For the successful navigation, mobile robots must robustly carry out a few tasks such as obstacle detection and avoidance, path planning, map building and self-localization. Among them, self-localization, which is the estimation of its current location with respect to the immediate surroundings, is probably one of the most difficult functions[4].

Landmarks are divided into two classes: natural or artificial. The methods using natural landmarks are more general

Manuscript received Jul. 2, 2010; revised Dec. 6, 2010;

Accepted Dec. 7, 2010.

*** Corresponding Author : kjwook@dau.ac.kr

This study was supported by research funds from Dong-A University. approaches than those using artificial landmarks. Generally, natural landmarks are selected in the scene in consideration of their particular characteristics. Mobile robot learns those characteristics or keeps the features of the landmarks in memory and recognizes them using the neural network or some matching techniques while it moves [5, 6, 7]. Mosaic images of the outdoor environments are also used for an image matching based method [7].

In this paper, as a general approach of sensor fusion, a STSF (Space and Time Sensor Fusion) scheme is proposed for the joint and disjoint data structure and applied to the landmark identification and a mobile robot's navigation problem. This newly proposed STSF's effectiveness is shown by comparing the conventional sensor fusion with STSF and presenting the experimental results.

\section{Space and Time Sensor Fusion}

\subsection{General Pattern of Sensor Fusion}

Fig. 1 shows a general pattern of multi-sensor integration and fusion in a system. While the fusion of information tasks place at the nodes in the figure, the entire network structure together with the integration functions, shown as part of the system, are parts of the multi-sensor integration process.

Multi-sensor data fusion refers to the combination of sensory data from multiple sensors into a common representational format. The focus is on combination of data from various sensors into a single inferences or interpretation, without regard to how it is obtained, or how the sensors are organized. We assume that an appropriate sensing architecture and sensing plan has been devised to collect data. An example of a multiple 
sensor system that can support automatic object recognition(AOR) is shown in Figure 1[8,9].

For illustration, camera, ultrasonic, IR, and radar are shown. In this sensor -level fusion configuration, each sensor processes its data with algorithms that are tailored and optimized to the received frequency band, active or passive nature of the sensor, spatial resolution and scanning characteristics, object and background signatures, polarization information, etc. Results of the individual sensor processing are forwarded to a fusion processor where they are combined to produce a validated object or no-object decision[12].

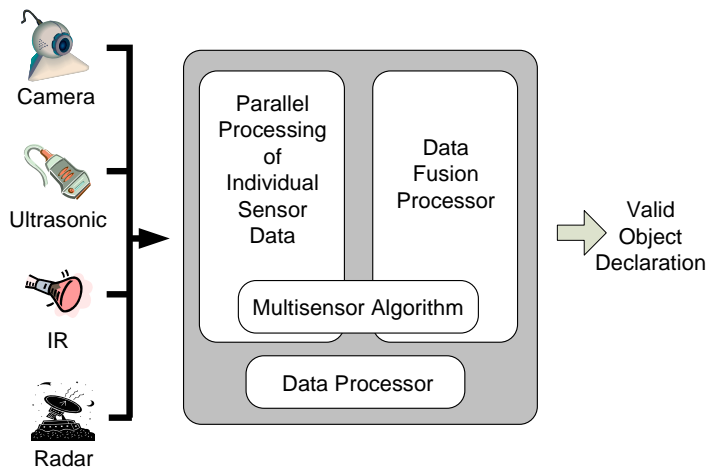

Fig. 1 Sensor fusion concept for ATR(automatic object recognition) using multiple sensor data.

One of the simplest and most intuitive sensor fusion is to take a weighted average of the measurements provided by a group of sensors and to use this as the fused value. While this method allows the real-time processing of dynamic low-level data in the most cases, a higher-level sensor fusion is required to achieve a reliable and accurate measurement in the unstructured environment. For the case, the Kalman filter is preferred because it provides a method that does not require heavy computation compared to the weighted average and results in the estimated fused measurements that are optimal in a statistical sense. A weighted average has been used for the multi-sensor fusion in the mobile robot navigation by Thrun[10], where the sensory information is preprocessed by the threshold operation to eliminate spurious measurements.

Let us define the $k^{\text {th }}$ moment data provided by $i^{\text {th }}$ sensor as, $z_{i}(k)$, which is transformed into a measurement, $x_{i}(k)$. Then the conventional sensor fusion, that is, a spatial fusion technique provides the measurement as

$$
\hat{x}(k)=\sum_{i=1}^{n} W_{i} x_{i}(k)
$$

wher $\sum_{i=1}^{n} W_{i}=1, \quad x_{i}(k)=H_{i} z_{i}(k) \in R^{m}, n$ is the number of sensors, $H_{i}$ represents a transformation from the sensory data to the $m$ dimensional measurement vector, and $W_{i} \in R^{m \times m}$ represents the weight for $i$-th sensor.

Note that in obtaining $z_{i}(k)$, the low-level fusion might be applied with multiple sets of data with known statistics [1,2]. The transformation $H_{i}$ is purely dependent on the sensor type and the decision of $W_{i}$ can be done through the sensor fusion process. Later these data are provided to the linear model of the control/measurement system as current state vector, $x(k)$.

\subsection{Use of Spectral Estimation Techniques}

The STSF (Space and Time Sensor Fusion) scheme combines the sensory information acquired at different instants from different sensors to determine the measurement. It may expend its applicability to the systems where the states at each instant can be predicted as shown in Fig. 2.

Estimation of parameter block may provide the measurement vector at each sampling moment. The blocks of verify the significance and adjust weight are pre-processing stages for the sensor fusion.

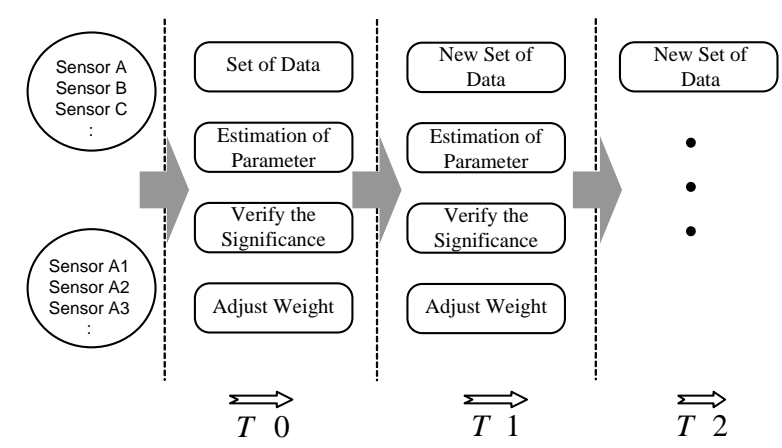

Fig. 2 Data processing for STST.

After these steps, the previous data set will be fused with the current data set, which provides a reliable and accurate data set as the result of multi-sensor temporal fusion. In the figure, the significance implies that how much the previous data set is related to the current data. An arbitrary value of significance may cause the problem to be complex. Therefore, some people may consider whether it corresponds to the same data or not, that is, 1 or 0 . When the significance is 0 , the weight can be adjusted simply to 0 . However, when the significance equals 1 , the adjustment of weight should be properly performed to provide reliable and accurate data. In the next sub-section, we will introduce a simple methodology for the weight adjustment and significance decision.

The STSF can be represented mathematically as follows:

$$
\hat{x}(k)=\sum_{i=1}^{n} W_{i}\left\{\sum_{j=1}^{k} P_{j} T S_{i}(j)\right\}
$$

where $\sum_{j=1}^{k} P_{j}=1$.

Note that when each of sensor information can provide the measurement vector, that is, the redundant case $T S_{i}(j)$ can be expanded as, 


$$
T S_{i}(j)=T_{i j}+H_{i} Z_{i}(j)
$$

where $T_{i j}$ represents the homogeneous transformation from the location of the $j^{\text {th }}$ measurement to the $i^{\text {th }}$ measurement.

However, when the multi-sensors are utilized in the complementary mode, the transformation relationship cannot be defined uniquely; instead it will be defined depending on the data constructing algorithm from the measurements. For an example, a single image frame captured by a camera on a mobile robot cannot provide the distance to an object until the corresponding object image is provided from a different location.

Each previous data set is transformed to the $k^{\text {th }}$ (current) sampling location, and represented by the measurement vector, $T S_{i}(j)$. Now how can we fuse the $k$ data sets into a reliable and accurate data set? In the Eq. (2), $W_{i}$ can be determined by the geometrical relationship among sensors, in other words, by the spatial sensor fusion considering the noise. While the image frame is tracking a feature, the sensor generates a stream of measurements. In analyzing the measurements from the sensor, the greyvalues $g$ and image $f$ over time can be treated as random processes. If there is no relative motion between the feature being tracked and the sensor, then the random processes can be viewed as stationary. When there is a relative motion between the feature and the sensor, the processes then cease to be stationary. As an illustration of low-level sensor fusion, we shall only consider the stationary case. In order words, we assume that there is no motion between the sensor and the feature being tracked in the image. The interest in the proceeding analysis lies only in determining whether the process noise is white or not.

Determination of the significance $P_{j}$ is the final step for the temporal sensor fusion. Note that this expands the dimension of sensor fusion from one to two. As one of solid candidate, we propose here to use the auto-correlation as an index for the significance adjustment and have the form,

$$
\Psi_{j}=\sum_{k=-\infty}^{\infty} T S_{i}(k) T S_{i}(j+k) .
$$

Depending on the correlation, $P_{j}$ will be determined as

$$
P_{j}=\frac{\Psi_{j}}{\sum_{j=1}^{k} \Psi_{j}}
$$

\section{Experiments}

In this STSF approach, the data obtained by the sensors are utilized until they do not have any efficiency for the measurement decision. The data set can be either redundant to improve the accuracy or complementary for the complete measurement. For the latter case, this STSF scheme is essential for the measurement. For an example, a single camera system is not capable of measuring the distance to an object. However, if we utilize this STSF scheme, the distance measurement is possible by only the single camera. Note that human keeps the image only about $1 / 15$ seconds on his retina and he cannot measure the distance by the single eye. However the robot is capable of keeping the image for a couple of hours. This enables a single camera to take over the function of stereo camera system that measures the distance using the images captured by the two cameras separated by $d \mathrm{Cm}$.

\subsection{Landmark Design for Robust Detection}

The proposed landmark is made of square patterns with symmetric and repetitive arrangement of black and white patches as shown in Fig. 3. Such arrangement of black and white patterns in the landmark is robust against geometric distortions in indoor environments. Even if the landmark rotates and changes its scale in the image frame, grey histogram and its projection characteristics do not change very much.

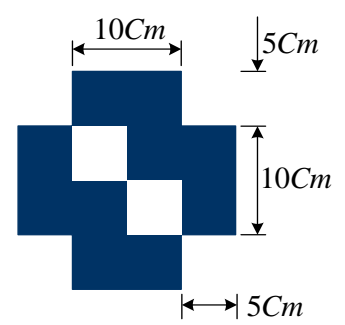

Fig. 3 Landmark pattern and size.

To recognize the landmarks, this paper describes a system for landmark tracking by a template matching approach. An adaptive template matching approach based on a Bayesian decision technique [11] is used for self-localization.

As a measure, how well an arbitrary pattern of greyvalues, a template $g(m, n)$, matches to a given image $f(i, j)$, the distance function, $d$, is defined as,

$$
d=\int_{G}(f-g)^{2} \text { or } \max _{G}|f-g|
$$

The minima of these measures imply the best match. In the discrete case, this takes the form as,

$$
M(i, j)=\sum_{m} \sum_{n} f(i+m, j+n) \cdot g(m, n),
$$

where the maximum of $M(i, j)$ corresponds to the best match. This "cross-correlation”' yields a result only if the integral is computed over the whole area $G$.

\subsection{Landmark Recognition}

The mobile robot with CCD camera is commanded to follow the environment as shown from (a) to (b) of Figure 4. We performed the experiment for two cases.

To begin with, the 2-D landmark used by experiments is 
shown in Figure 4. The primary pattern of landmark is a $5 \mathrm{~cm}$ white square block on black background and a $5 \mathrm{~cm}$ square block. The major reasons for choosing the square blocks are

- The projection of a square block in the image plane can always be approximated by an ellipse, which is easy to recognize using the elliptical Hough transformation technique.

- A circular pattern does not get mixed up easily with the majority of patterns frequently seen in an indoor environment, such as circular disk, polygonal objects, etc.

- A square pattern is more robust to noise and occlusion than circular, polygonal patterns during template matching process, even though all these patterns can be detected by using Hough transformation technique[13].

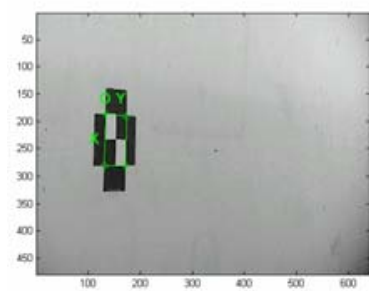

(a)

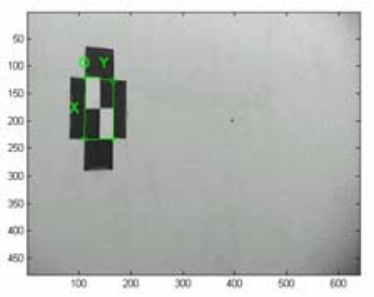

(b)
Fig 4. Landmark images detected by the camera.

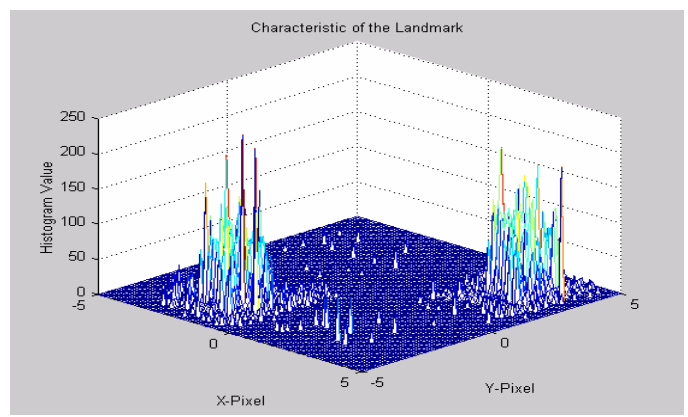

(a) normal

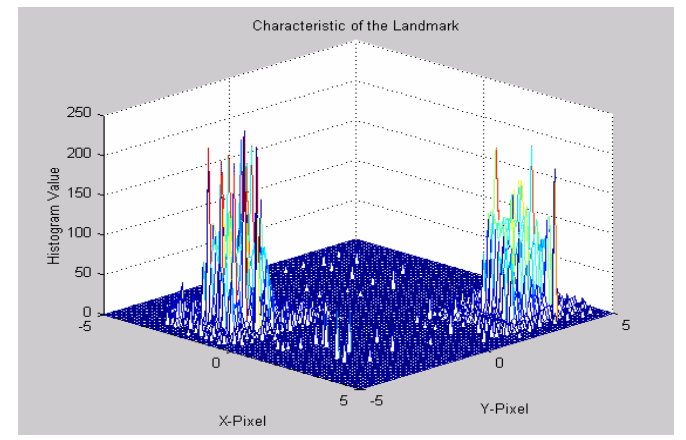

(b) rotating

Fig 5. Global histograms of the landmark under the geometrical variations.

To detect the landmark in the cluttered scene robustly, the landmark should show invariant characteristics under some distortions. Invariance under geometric distortions is related to the shape and gray pattern of the landmark and invariance under the photometric distortions is related to the size and shape in which processing is preformed. Shape and gray pattern of the landmark is also important for the robust detection and tracking.

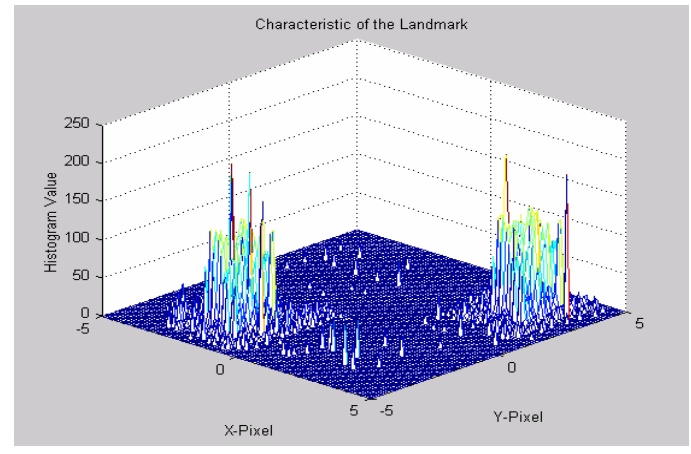

(a) normal

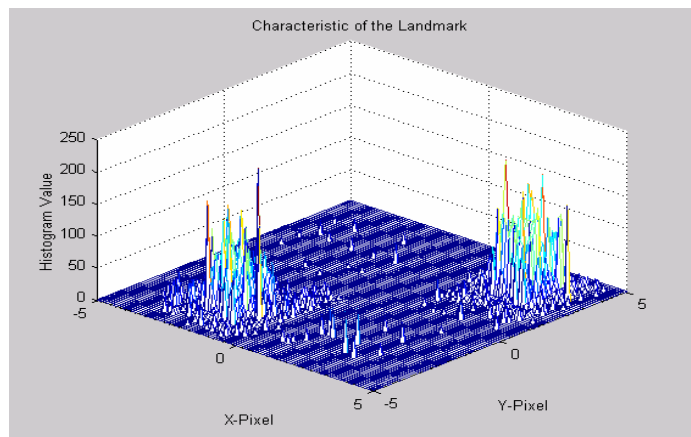

(b) zooming

Fig 6. Projected histograms of the landmark under the geometrical variations.

The proposed landmarks are made of gray patterns with symmetric and repetitive arrangement of gray patches as shown in Fig. 5. Such arrangement of gray pattern in the landmark is robust against geometric distortions in indoor environments. Even if the landmark rotates and changes its scale in an image, gray histogram and its projection characteristics do not change very much. Fig. 5 and Fig. 6 show these characteristics of the landmark under geometric distortions [7, 14].

$$
\left[\begin{array}{ll}
b & w
\end{array}\right]=\left[\begin{array}{ll}
\frac{b}{b+w} & \frac{w}{b+w}
\end{array}\right]
$$

To make each landmark distinguishable from the other, gray colors having maximum distance by template matching in the chromaticity gray space are selected for each landmark. We compute a symmetric type of gray histograms of the landmark as the key features for detection and racking.

Global histogram is knows to represent the global gray characteristics. Projected histogram conveys the local gray property of the landmark. A small number of rows and columns are selected to reduce the computational complexity of projected histogram. Fig. 5 and Fig. 6 show that the global histogram and projected histogram are almost invariant under the variations. 


\section{Conclusions}

In this paper, a new sensor fusion concept, STSF (Space and Time Sensor Fusion), was introduced. The effectiveness of STSF was demonstrated through the preliminary robust landmark detection experiments. To follow the navigation trajectories without $a$ prior information on the environment, not only the data from the sensors located at different places but also the previous sensor data are inevitably necessary. This scheme may require more memory space and computing power in the navigation system.

With the tracked single landmark, we successfully obtained the absolute location of the robot. Experimental results show that the proposed landmark model is effective and can be detected and tracked in a cluttered scene robustly. Based on these results, further experiments will aim at applying the STSF to the control of a mobile robot in an unstructured environment with various sensors.

\section{References}

[1] Ren C. Ruo and Kuo L. Su, "A Review of High-level Multisensor Fusion: Approaches and applications,” Proc. of IEEE Int'l. Conf. On Multisensor Fusion and Integration for Intelligent Systems, pp. 25-31, Taipei, Taiwan, 1999.

[2] P. Weckesser and R. Dillman, "Navigating a Mobile ServiceRobot in a Natural Environment Using Sensor-Fusion Techniques," Proc. of IROS, pp.1423-1428, 1997.

[3] Luo, R.C., Chih-Chen Yih,and Kuo Lan Su "Multisensor fusion and integration: approaches, applications, and future research directions,” Sensors Journal, IEEE, Vol. 2 Issue. 2, p. 107-119, April 2002.

[4] A. J. Briggs, D. Scharstein, D. Braziunas, C. Dima, P.Wall, “Mobile Robot Navigation Using Self-Similar Landmarks," In Proc. of IEEE International Conference on Robotics and Automation, pp. 1428-1434, 2000.

[5] C. F. Olson, "Probabilistic self-localization for mobile robots", IEEE Transactions on Robotics and Automation, vol. 16 1, pp. 55-66, Feb. 2000.

[6] E. B.Meier, F. Ade, "Using the condensation algorithm to implement tracking for mobile robots," Third European Workshop on Advanced Mobile Robots(Eurobot '99), pp. 7380, 1999.

[7] K. J. Yoon, I. S. Kweon, C. H. Lee, J. K. Oh, I. T. Yeo, "Landmark design and real-time landmark tracking using color histogram for mobile robot localization," In Proc. of the 32nd International Symposium on Robotics, pp. 1083-1088, 2001.

[8] TaeSeok Jin, JangMyung Lee, and S.K. Tso, "A New Approach Using a Sensor Data Fusion for a Mobile Robot Navigation” ROBOTICA, Vol. 22, No. 1, pp. 51-59, 2004.

[9] X. Zhau, Q. Luo, B. Han, "Survey on robot multi-sensor information fusion technology,” Intelligent control and automation(WCICA2008), pp. 5019-5023, 2008.

[10]W. Pieczynski, "Unsupervised Dempster-Shafer fusion of dependent sensors,” in Proc. IEEE Southwest Symp. Image Analysis and Interpretation (SSIAI'2000), Austin, TX, Apr. 24, pp. 247-251, 2000.

[11] Ayala, V. Hayet, J.B. Lerasle, F. Devy, M., "Visual localization of a mobile robot in indoor environments using planar landmarks," Intelligent Robots and Systems, (IROS 2000). Proc. of IEEE/RSJ International Conference on, vol. 1, pp. 275-280, 2000.

[12] J. M. Richardson and K. A. Marsh. "Fusion of multisensor data," International Journal of Robotics Research, 7(6): pp. 78-96, 1988.

[13]D. L. Hall and j. Llinas, "A challenge for the data fusion community I: Research imperatives for improved processing," in Proc. $7^{\text {th }}$ Natl. Symp. On Sensor Fusion, Albuquerque, NM, Mar. 1994.

[14]D. Klimentjew, N. Hendrich, J. Zhang, "Multi sensor fusion of camera and 3D laser range finder for object recognition,” 2010 IEEE Conference on Multisensor Fusion and Integration for Intelligent Systems (MFI2010), pp. 236 - 241, 2010.

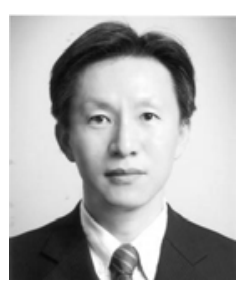

Tae-Seok Jin

He received the Ph.D. degrees from Pusan National University, Busan, Korea, in 2003, in electronics engineering.

He is currently an Assistant Professor at DongSeo University. From 2004 to 2005, he was a Postdoctoral Researcher at the Institute of Industrial Science, The University of Tokyo, Japan. His research interests include network sensors fusion, mobile robots, computer vision, and intelligent control. Dr. Jin is a Member of the KIIS, ICROS, and RSJ.

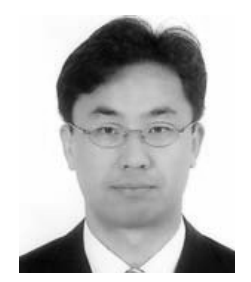

\section{Hyun-Sik Kim}

He received the B.S., M.S., and Ph.D. degrees in Electrical Engineering from Pusan National University, Korea in 1994, 1996, and 2001, respectively. He had been a Senior Researcher at the Agency for Defense Development. He is now an Assistant Professor of Dept. of Robot System Engineering in Tongmyong University. His research interests include intelligent control, evolutionary algorithms, robotics, underwater vehicle control, target tracking, and underwater target classification.

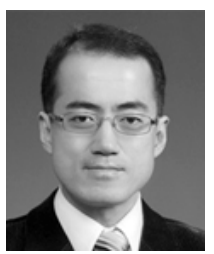

Jong-Wook Kim

He received the B.S., M.S., and Ph.D. degrees from the Department of Electronics and Electrical Engineering at Pohang University of Science and Technology 
International Journal of Fuzzy Logic and Intelligent Systems, vol. 10, no. 4, December 2010

(POSTECH), Pohang, Korea, in 1998, 2000, and 2004, respectively. Currently, he is an Assistant Professor in the Department of Electronics Engineering at Dong-A University, Busan, Korea.

His current research interests are numerical optimization methods, robot control, intelligent control, diagnosis of electrical systems, and system identification. 